\title{
Relocation of nucleolar fibrillarin in Trypanosoma cruzi during stationary phase
}

\author{
ERNESTO GUERRERO-SÁNCHEZ ${ }^{1}$, ANA MARÍA CEVALLOS ${ }^{1}$, \\ SANTIAGO MARTÍNEZ-CALVILLO ${ }^{2}$, IMELDA LÓPEZ-VILLASEÑOR ${ }^{1}$ and \\ ROBERTO HERNÁNDEZ ${ }^{1} *$ \\ ${ }^{1}$ Departamento de Biología Molecular y Biotecnología, Instituto de Investigaciones Biomédicas, Universidad Nacional \\ Autónoma de México, Ciudad Universitaria, CP 04360, México \\ ${ }^{2}$ Unidad de Biomedicina, Facultad de Estudios Superiores Iztacala, Universidad Nacional Autónoma de México, Av. De \\ los Barrios 1, CP 54090, México
}

(Received 13 Fuly 2015; revised 9 September 2015; accepted 15 September 2015)

\section{SUMMARY}

Trypanosoma cruzi is the aetiological agent of Chagas disease. Our group has focused on the study of ribosomal RNA and nucleolar structure in this organism. In this work, we address the cellular location of fibrillarin in epimastigotes. As a conserved and unreported feature in trypanosomatids, fibrillarin in T. cruzi is encoded by two genes that differ by approximately $35 \%$ in their deduced amino acid sequences (TcFib 1 and TcFib 2). Chimaeric fluorescent versions of TcFib1 and TcFib2 were individually expressed in T. cruzi cells. Both transfected cultures showed cells with a nucleolar fluorescent signal. We have not found any evident distinction between the structure or expression of Tcfibrillarins to propose a functional difference in cells. With the aid of an anti-TcFib 2 antibody, it was found that the endogenous protein relocates outside of the nucleolus in stationary epimastigotes. This was also the case in metacyclic trypomastigotes observed from aged cultures. The significance of this observation is not known, but a deficiency of fibrillarin nucleolar retention correlates with the observed reduction in the abundance of the pre-ribosomal RNAs species at stationary phase, and suggests that the nucleolar location of this protein depends on physiological processes.

Key words: fibrillarin, nucleolus, kinetoplastid stationary phase.

\section{INTRODUCTION}

Trypanosoma cruzi is a parasitic protozoan causative of Chagas disease, a neglected condition that causes an important health problem in Latin America. Our research group has been interested in ribosomal RNAs (rRNAs) and the biogenesis of ribosomes in this organism (Nepomuceno-Mejía et al. 2010; Hernández and Cevallos, 2014). Eukaryotic rRNA is synthesized in the nucleolus by RNA polymerase I as a precursor transcript that maturates by a complex molecular pathway. Throughout this process, the pre-rRNA is cut by internal cleavages and modified at specific positions. The main modifications of this fundamental transcript are pseudouridylation and methylation of the $2^{\prime}$ carbon of the ribose sugar ring (2'-O-methylation). Small nucleolar RNAs (snoRNAs) specifically interact (basepairs) with sequences on the pre-rRNA and guide these modifications. Furthermore, some snoRNAs are required for the cleavage events that release transcribed rRNA spacers (Gerbi et al. 2001). rRNA genes in trypanosomes are atypical in that a complex processing pathway leads to a

* Corresponding author: Departamento de Biología Molecular y Biotecnología, Instituto de Investigaciones Biomédicas, Universidad Nacional Autónoma de México, Ciudad Universitaria, CP 04360, México. E-mail: robertohf@biomedicas.unam.mx discontinuous large subunit rRNA component composed of two large and five small rRNAs (see Hernández and Cevallos, 2014, for a recent review). The African species of trypanosomes, Trypanosoma brucei, is the best-studied species among kinetoplastids. It has been reported that some of their snoRNAs are conserved among eukaryotes, while some others are species-specific (Michaeli, 2012), as expected for its atypical prerRNA cleavage pathway. snoRNAs occur in eukaryotic cells as molecular complexes with proteins (snRNPs). In the case of snoRNAs from the C/D family (i.e. RNAs that contain the conserved sequence motifs of Box C and Box D, Gerbi et al. 2001), four proteins have been identified: Fibrillarin (yeast Nop1p), Nop56p, Nop58p and Snu13p (Lafontaine and Tollervey, 2000). The former contains a reported methyltransferase catalytic domain (Me'Tase) involved in the 2'-Omethylation modification of pre-rRNA referred to above. Eukaryotic fibrillarins contain two distinct domains, an N-terminal glycine- and arginine-rich domain (GAR domain) (Amiri, 1994) and a larger catalytic domain MeTase. The catalytic domain of all fibrillarins is highly conserved, consisting of a central seven-stranded $\beta$-sheet surrounded by three $\alpha$-helices on each side. This structure is similar to that found in AdoMet-dependent Me'Tases (Amiri, 1994; Wang et al. 2000). Importantly, fibrillarin is 
essential for cell growth (Schimmang et al. 1989), and its depletion in yeast cells affects all pre-rRNA processing reactions and impairs pre-rRNA methylation (Tollervey et al. 1991). Similarly, fibrillarin from $T$. brucei is essential because silencing of the homologue NOP1 by RNA interference produces rRNA-processing and modification defects that lead to cell death (Barth et al. 2008). It is to be noted that fibrillarin gene annotation in this species shows the occurrence of three genes, but this situation has not been reported in the literature (Barth et al. 2008). As mentioned above, we are interested in T. cruzi ribosome biogenesis. Consequently, construction and characterization of nucleolar markers in this species represents a rational approach to follow the process. Fibrillarin has been reported as a nucleolar marker of the dense fibrillar component (Olson and Dundr, 2005). In this work, it is shown that plasmid-encoded fluorescent fusions of two dissimilar fibrillarins in T. cruzi are expressed as nucleolar proteins and, in at least one case (here called TcFib2), the native endogenous protein is relocated outside the nucleus in a population of cultured epimastigotes at stationary phase. So is the case of metacyclic trypomastigotes, which are non-dividing forms devoid of nucleoli (Elias et al. 2001).

\section{MATERIALS AND METHODS}

\section{Parasites and culture conditions}

Trypanosoma cruzi epimastigotes from the CL Brener strain were grown at $28^{\circ} \mathrm{C}$ in liver infusion tryptone medium supplemented with $10 \%$ heatinactivated fetal bovine serum (Camargo, 1964). For growth curves, cultures were seeded at a density of $1 \times 10^{6}$ cells $\mathrm{mL}^{-1}$ and counted at 24-48 $\mathrm{h}$ intervals. Cells were harvested during exponential growth (cells duplicating every $24 \mathrm{~h}$; cellular density of approximately $8-12 \times 10^{6}$ cells $\mathrm{mL}^{-1}$ ) or during stationary phase [when parasites stopped their growth for $72 \mathrm{~h}$; cellular density of $90-100 \times 10^{6}$ cells $\mathrm{mL}^{-1} ; 12-14$ days post inoculation (dpi)].

\section{Plasmid construction}

Trypanosoma cruzi genomic DNA was prepared from exponentially growing epimastogtes by standard procedures. The experimentally determined coding regions of TcFib1 and TcFib2 of the $\mathrm{S}$ allele were amplified from genomic DNA and cloned into the $E c o$ RI site of the vector pGEX-3X to be expressed as GST-fusion proteins (GE Healthcare). These coding regions were also amplified and cloned without the stop codon between the EcoRI and Cla I sites present in the pTEX vector (Kelly et al. 1992). Downstream of these genes and in frame with them, the coding region of the EGFP from the
pRH006 vector (BCCM/LMBP, TechnologieparkZwijnaarde, Belgium) was subcloned into the Cla I-Xho I sites of the pTEX vector. All plasmid inserts were sequenced. When transfected into epimastigotes, the plasmids pTEX-TcFib1-EGFP and pTEX-TcFib2-EGFP would express fusion proteins with EGFP in the C-terminal region. Trypanosoma $c r u z i$ epimastigotes were transfected with the indicated plasmids as earlier described (MartínezCalvillo et al. 1997).

\section{Northern blot analysis}

For Northern blot analysis, total RNA was isolated from epimastigotes using TRIZOL ${ }^{\circledR}$ reagent. RNA was electrophoresed in $1 \%$ agarose gels with $2 \mathrm{M}$ formaldehyde, $1 \mathrm{~mm}$ ethylenediaminetetraacetic acid (EDTA) and $50 \mathrm{~mm}$ MOPS buffer, pH 7.0. Blot transfers and hybridizations were performed under standard conditions. The hybridization patterns of three rRNA gene probes, which span most of the rRNA cistron, were evaluated in both exponential and stationary phases of growth (Hernández et al. 1988).

\section{Expression and purification of recombinant GST- fibrillarin fusion proteins}

GST-TcFib1 and GST-TcFib2 were expressed in Escherichia coli strain BL21 (DE3) and the recombinant proteins were affinity purified using a glutathione Sepharose $4 \mathrm{~B}$ column according to the manufacturer's instructions (Amersham Biosciences, London, UK). To remove contaminants, the proteins eluted from the column were separated by $10 \%$ sodium dodecyl sulphate-polyacrylamide gel electrophoresis (SDS-PAGE) and stained with Coomassie Brilliant Blue, and the band corresponding to the recombinant fusion protein was cut from the gel and electroeluted. The electroeluted protein was dialysed overnight at $4{ }^{\circ} \mathrm{C}$ in $10 \mathrm{~mm}$ Tris- $\mathrm{HCl}$, $\mathrm{pH} 8,100 \mathrm{~mm} \mathrm{NaCl}$ and $10 \%$ glycerol. The protein was then concentrated by ultra filtration in Centricon 30 filter units (Millipore Corporation, Bedford, USA) and stored at $-20^{\circ} \mathrm{C}$ until use.

\section{Production of mouse anti-T. cruzi fibrillarin polyclonal antibodies}

Ten micrograms of either GST-TcFib1 or GSTTcFib2 were emulsified with Freund's complete adjuvant and administered subcutaneously to BALB/c mice $(200 \mu \mathrm{L}$ per mice). Two booster doses $(10 \mu \mathrm{g}$ of protein emulsified in incomplete Freund's adjuvant per booster dose, subcutaneous) were administered 9 and 7 weeks after the previous immunization. Pre-immune blood was collected. Fifteen days after the last antigen boost, blood was collected by terminal cardiac puncture. Serum was obtained from 
all samples, aliquoted and stored at $-70{ }^{\circ} \mathrm{C}$ until use. The immunization protocol and housing conditions were approved by the local Bioethics Committee for Animal Research.

\section{Immunoblotting}

Total parasite lysates were separated by $10 \%$ SDSPAGE and transferred to a $0.45 \mu \mathrm{M}$ polyvinylidene difluoride Immobilon-P Membrane (Merk Millipore, Darmstadt, Germany) according to the standard protocols. Nitrocellulose membranes were blocked with $0.05 \%$ Triton $\mathrm{X}-100$ in phosphate buffered saline (PBS-T) with $5 \%$ skim milk overnight at $4{ }^{\circ} \mathrm{C}$. The membrane was then incubated for $1 \mathrm{~h}$ at room temperature (RT) with anti-TcFib serum diluted in PBS-T with $2 \%$ skim milk. At the end of the incubation period, the membrane was washed three times with PBS-T (30 min per wash) and then incubated for $1 \mathrm{~h}$ with horseradish peroxidase-labelled goat anti-mouse IgG (ImmunoPure, Pierce) diluted in PBS-T with $2 \%$ skim milk (1:30 $000, \mathrm{v} / \mathrm{v})$. The membrane was washed three times with PBS-T (3 min per wash), incubated in SuperSignal West Pico Chemiluminescent Substrate (Pierce), and then exposed to film and developed. Polyclonal sera were screened by immunoblotting of $T$. cruzi protein lysates. Three anti-TcFib2 sera recognized a band of the expected size $(\sim 30 \mathrm{kDa})$, whereas none of the anti-TcFib1 sera recognized any $T$. cruzi antigen. One of the anti-TcFib2 sera was used in this study at a dilution of 1:6000 in immunoblots. In some studies, polyclonal antibodies against $\mathrm{Tc}$ actin were used at a dilution of 1:8000 (Cevallos et al. 2011).

\section{Fluorescence microscopy}

For immunofluorescence studies, epimastigotes from both exponential and stationary phases of growth were washed two times for 5 min each with PBS, resuspended in $4 \%(\mathrm{w} / \mathrm{v})$ paraformaldehyde in PBS $\left(20 \times 10^{6}\right.$ cells $\left.\mathrm{mL}^{-1}\right)$, and allowed to adhere to Saline-Prep Slides (Sigma-Aldrich, Saint Louis, Missouri, USA) for $10 \mathrm{~min}$ at RT. Fixed parasites were permeabilized with $0 \cdot 5 \% \quad(\mathrm{v} / \mathrm{v})$ Triton X-100 for $5 \mathrm{~min}$, and then blocked with 5\% $(\mathrm{w} / \mathrm{v})$ bovine serum albumin in PBS for $2 \mathrm{~h}$. The blocked cells were first incubated with primary antibodies (1:400 dilution in 1\% BSA in PBS) for $1 \mathrm{~h}$ at RT, and then labelled with donkey anti-mouse IgG $(\mathrm{H}+\mathrm{L})$ tagged with Alexa Fluor 488 (1:400 dilution) for $1 \mathrm{~h}$ at RT in the dark. Negative control samples were incubated with pre-immune mouse serum. For the analysis of EGFP fluorescence in epimastigotes, transfectant cells were washed, fixed and allowed to bind to Saline-Prep Slides with the same protocol described above. Then, the slides were washed with PBS to remove non-adherent cells.
Both types of preparations were mounted for fluorescence in Vectashield medium containing diamidino-2-phenylindole (DAPI) to stain nucleic acids (Vectashield with DAPI; Vector Laboratories, Burlingame, California, USA). The samples were observed with an epifluorescent Olympus BX51 microscope, and the images were obtained with an Olympus camera and analysed using Image J (Collins, 2007).

\section{Sequence alignment analysis}

Homologous deduced T. cruzi fibrillarin protein sequences were obtained from the TriTrypDB database (http://tritrypdb.org/tritryp/) and submitted to the Clustal Omega web server (Sievers et al. 2011; http://www.ebi.ac.uk/Tools/msa/clustalo/). The Jalview applet allowed us to view, edit, colour and output the alignment for insights into amino acid conservation using the Blossum62 matrix parameters (Waterhouse et al. 2009).

RESULTS

\section{Two coding genes for fibrillarin in T. cruzi}

The Tri'TrypDB genomic resource (version 8.0) was searched for genes encoding fibrillarins in the T. cruzi hybrid strain CL Brener (El-Sayed et al. 2005). Esmeraldo-like (S) and non-Esmeraldo like (P) are the terms used to name both gene haplotypes. Four putative fibrillarin alleles were identified in this genome database at two loci (chromosome 4 and chromosome 40). Both allelic variants in each locus (S and $\mathrm{P}$ ) are clearly annotated (Fig. 1A). Herewith, the proteins encoded at chromosome 4 have been named fibrillarin 1 (TcFib1) and those encoded at chromosome 40 as fibrillarin 2 (TcFib2). As originally annotated, the four fibrillarin genes (two pairs of allelic genes) differ in size: TcFib1-S (TcCLB.510105.50) is $915 \mathrm{bp}$ long, TcFib1-P (TcCLB.509715.40) is $951 \mathrm{bp}$, TcFib2-S (TcCLB.506211.250) is $879 \mathrm{bp}$ and TcFib2-P (TcCLB.511287.150) is $876 \mathrm{bp}$. Moreover, analysis of the aligned sequences showed a 5 '-end terminal extension on both haplotypes of TcFib1 (Fig. 1B). To determine whether this extension is actually present in the mature mRNAs, their trans-splicing site was experimentally determined. For both haplotypes, the TcFib1 mRNA trans-splicing site was actually located downstream of the originally annotated translation starting ATG (Fig. 1B). Consequently, the gene length of both TcFib1 haplotypes becomes the same as that of TcFib2-P. To further corroborate the annotated genes as fibrillarins, the deduced protein sequences were evaluated by the Conserved Domain Database at NCBI (Finn et al. 2014). In all cases, the pfam motif 01269 'fibrillarin' was identified. 
A

\begin{tabular}{|cccccccc|}
\hline Name & Chromosome & Haplotype $^{\star}$ & Gene ID & \# bp & \#aa & kDa & pl \\
TcFib1 & 4 & S & TcCLB.510105.50 & 876 & 291 & 30.95 & 10.00 \\
& & P & TcCLB.509715.40 & 876 & 291 & 30.92 & 9.97 \\
TcFib2 & \multirow{2}{*}{40} & S & TcCLB.506211.250 & 879 & 292 & 30.56 & 9.90 \\
& & P & TcCLB.511287.150 & 876 & 291 & 30.48 & 9.81 \\
\hline
\end{tabular}

${ }^{*}$ S Esmeraldo-like P Non-Esmeraldo like

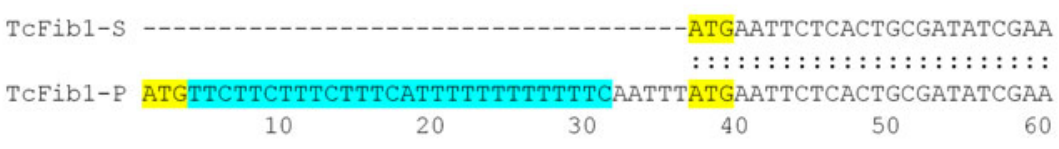

30
50

60

70

TCFib1-S TTTAAGAATAGCGCAATGCGTGGAGGATTCGGTCGTGGAGGGTTTCGCGGTGGTGGAAGA $::::::::::::::::::::::::::::::::::::::::::::::::::::::::::::::::::::$ :

TCFib1-P TTTAAGAATAGCGCAATGCGTGGAGGATTCGGTCGTGGAGGGTTTCGCGGTGGTGGAAGA

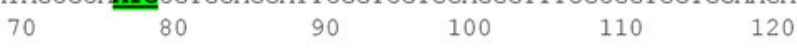

C

TcFibl-S

TcFib1-P

TcFib2-S

TCFib2-P

\section{TCFib1-S \\ TCFib1-P \\ TCFib2-S \\ TCFib2-P}

\section{Glycine and Arginine Region \\ 30}

10

20

40

50

MRGGFGRGGFR- - GGGRGGG SSARGRGGA SRGAGRGGPGRGGRGGRGGG Á AKV- - - - 53 NRGGFGRGGFR--GGGRGGG SSARGRGGA SRGAGRGGPGRGGRGGRGGGAGAKV_... MRGGRGGGGRGGRGGGRGGGR--GGGRGGGRGGGRGGGGGRGGGGRGGGRGGSSAVRGNV 58 IRGGRGGGRGGGRGGGGRGG--- GGRGGGGRGGGGRGGGGRGGGGRGGGRGGSSAVRGNV 57

\section{Methyltransferase Domain}

$\begin{array}{lccc}70 & 80 & 90 & 100\end{array}$

VEPHMLHPGVFISKGKADSLCZLNMVPGVSVYGEKRIELGAZQGGDEKKEYRLWNPYRSK 113 VEPHM LHPGVF ISKGKAD S LC I LN VPGV SVYGEKR IELGA ZQGGDEKKEYRLW NPYR SK 113

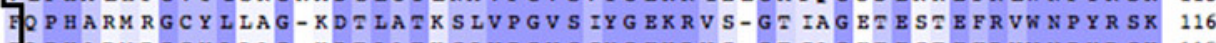
RPBARMRGCYLLAG-KDILAIKSLVPGVSIYGEKRVS-GFIAGEIESTEFRVWNPYRSK 115

130

140

150

160

170

TCFib1-S TCFib1-P TCFib2-S TCFib2-P LAAA IYAGVGSIHM K PG SKVIYLGGASGETVSHVSDLVGPEGMVYAIEFSNRSGRDLVDM 173

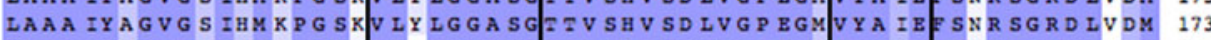
LAAA IY SGVAQ IYM EPG SSVISLGAA SGI ZVSHVSDLVGPEGVVYAVEFSHR SGRDLEEM 176 LAAA IYSGVAQ IYM EPGS SULYGAASGI ZVSHVSDLVGPEGVVYAVEFSHRSGRDLEEM 175 LAAA IYSGVAC IYMEPGSSELYLGA

190

200

210

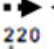
230

TCFib1-S

TCFib1-P

TCFib2-S

TcFib2-P

SKRRPN IVP IIEDARYPM KYRM LVP-MVDCVFMDVAQPDQAR ILALNAQAFLKNGGHYVI 232 SKRRP N IVP IIEDARYPM KYRM LVP MVDCVFNDVAQPDQAR ILA LNAQAFLKNGGHYV I 232 TKRRN IVP ILEDARYPQKYRM I IPR LVDC IFMDVAQPDQAR ILALNAQHFLKENGGFV I 236 I RRRN IVP ILEDARYPQKYRM I IPRLVDC IFMDVAQPDQAR ILALNAQHFLKENGGFV 235 $M$

TcFib1-S TcFib1-P TCFib2-S TCFib2-P

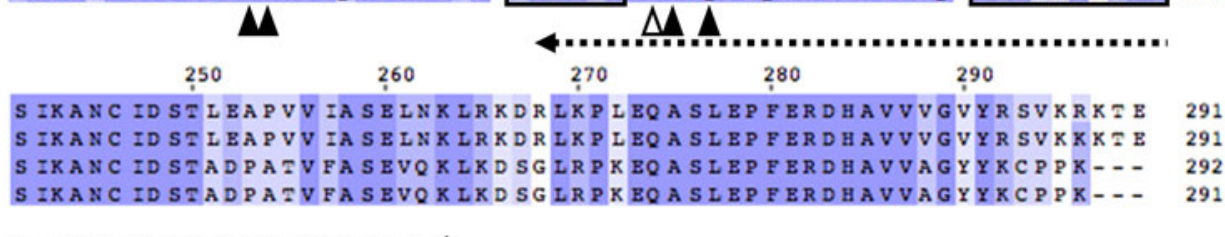

$\boldsymbol{\Delta}$ Interaction-stabilization S-Adenosyl Metionine

$\triangle$ Absolutely conserved aspartate

RNA Recognition Motif

Conserved motifs in Nop1p RNA folding octamer

Fig. 1. (A) Fibrillarin genes in the annotated T. cruzi genome, self-explained. (B) Alignment of the $5^{\prime}$ end of the putative coding regions of fibrillarins encoded on chromosome 4 (TcFib1). Highlighted in yellow are the ATG codons annotated in the genome database; in blue, a polypyrimidine-rich region potentially recognized in the trans-splicing process, and in green the demonstrated ATG leading the fibrillarin open reading frame. The trans-splicing site is in red lettering.

(C) Localization of conserved motifs in deduced T. cruzi fibrillarins. Sequence alignment of fibrillarin paralogues from T. cruzi was performed using Clustal Omega (Sievers et al. 2011; http://www.ebi.ac.uk/Tools/msa/clustalo/). Gaps and mismatches are colourless. Conserved residues are highlighted with blue. Colour intensity shows the level of conservation with respect to the Blosum62 matrix. Both GAR and MeTase domains are indicated. Graphic elements that mark conserved functional motifs and residues are explained at the bottom of the figure.

Sequence alignment (Clustal Omega) and comparisons of the predicted proteins from the annotated T. cruzi fibrillarin encoding genes indicate almost complete identity between haplotypes of the same gene (96-99\% identical residues), and modest conservation
(65-66\% identical residues) when the alignment comparison was carried out between the two deduced fibrillarin-type sequences (i.e. TcFib1 vs TcFib2).

Analysis of the deduced amino acid sequences of both T. cruzi fibrillarins allowed for the 
identification of relevant residues and motifs characterized in other species. Figure 1C depicts both the N-terminal GAR region and the MeTase domain. The MeTase domain initiates at residue V61 in TcFib1 and Q62 in TcFib2. A small collection of conserved residues was identified within the MeTase domain: Y142, G144, E168, F169, D193, A194, V195 and Q197. It has been shown from the archaebacterium species Aeropyrum pernix that these residues are involved in stabilizing the cofactor AdoMet (De Silva et al. 2012). In addition, the sequence data also permitted the identification of two potential RNA-binding sites (K120- G161 and V208- K264) and a conserved G M/V V Y A I/V $\mathrm{E} \mathrm{F}$ octamer that may promote the proper folding of the central RNA-binding site (Fig. 1C). These elements were previously characterized in a recombinant version of fibrillarin 2 from Arabidopsis thaliana (Rakitina et al. 2011). An absolutely conserved aspartate (D214 in our coordinates) has been distinguished with a central role in the catalytic function of eukaryotic and archaeal fibrillarins (Reichow et al. 2007). Finally, four functional motifs responsible for AdoMet binding in Saccharomyces cerevisiae can also be identified in the sequence here characterized: V140-G148; V164-E168; (M/L)207-F212; F231-I240 (Niewmierzycka and Clarke, 1999).

\section{Both recombinant fibrillarin genes are expressed and localized in the nucleolus}

Gene type specific fibrillarin DNA probes were hybridized with total RNA in Northern blots. It was found that both TcFib1 and TcFib2 genes are transcribed, and in the two cases RNA species of approximately $1.3 \mathrm{~kb}$ were revealed (not shown). This transcript size is more than sufficient to code for proteins of $31 \mathrm{kDa}$. To explore whether both fibrillarin encoding mRNAs produce nucleolar proteins, the two types of chimaeric fibrillarin-EGFP fusions were independently transfected into culturederived epimastigotes, and their cellular location was analysed by fluorescence microscopy. The occurrence of a fluorescent signal in the nucleolus was observed in cell lines transfected with the chimaeric gene of either type (i.e. TcFib1 or TcFib2). Transfections with a non-chimaeric EGFP bearing vector were analysed as a control, and its fluorescence was observed throughout the cell body (Fig. 2).

\section{Fibrillarin nucleolar relocation in stationary epimastigotes}

Growth-arrested epimastigotes at the stationary phase provide an experimental model in T. cruzi cells to address differences in nucleolar size and nucleolar architecture (Nepomuceno-Mejía et al. 2010). To analyse potential differences in the

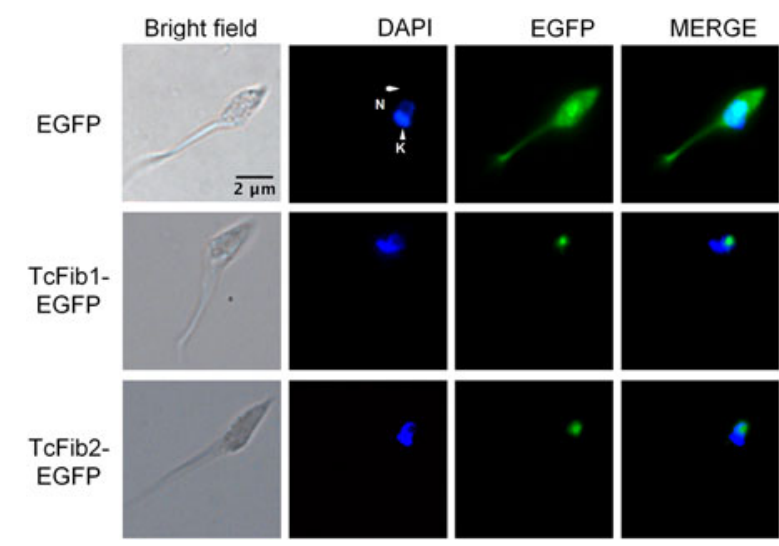

Fig. 2. Nucleolar expression of EGFP-tagged T. cruzi fibrillarins. Both T. cruzi fibrillarin genes were ectopically expressed as fusion proteins tagged with EGFP.

Transfection of the individual EGFP coding region is presented in the first row as a control. Nucleus and kinetoplastid organelles are indicated as $\mathrm{N}$ and $\mathrm{K}$, respectively.

abundance of fibrillarin between growing epimastigotes and stationary cells, anti-fibrillarin antibodies were prepared. Two groups of mice were immunized with either version of bacterial recombinant fibrillarin (i.e. GST-TcFib1 or GST-TcFib2), but it is to be noted that only the group of mice exposed to TcFib2 made informative antibodies (i.e. signal producers). Figure $3 \mathrm{~A}$ shows the recognition of the endogenous fibrillarin as well as of the fusion version of the protein by the anti-TcFib2 antibody. This antibody was used to probe Western blots with cellular extracts from exponential and stationary cells. Figure 3C illustrates a similar abundance of fibrillarin (at least TcFib2) in cells harvested at the referred different growing phases. Interestingly, changes in fibrillarin location within aged cells could be detected. A nucleolar concentration of the fluorescent signal was observed in epimastigotes from the exponential phase of growth, whereas in stationary cells the fluorescence was located throughout the cytoplasm, with a diffuse and granular pattern (Fig. 4). Conspicuously, the cytoplasmic relocation of fibrillarin in stationary cells could not be seen with the EGFP-tagged version of the protein. A structural hindrance of the chimaeric fibrillarin may be postulated to account for its defect in the nucleolar-cytoplasmic relocation event. Finally, in our culture conditions, approximately $10 \%$ of cells at the stationary phase transform into metacyclic trypomastigotes. These forms showed a granular cytoplasmic distribution of fibrillarin (Fig. 5).

An early observation of a partial fibrillarin relocation from the nucleolus to the cytoplasm was published for Xenopus kidney cells (Rivera-Léon and Gerbi, 1997). The experimental stimulus in this case was actinomycin $\mathrm{D}$, which is known to impair 
A

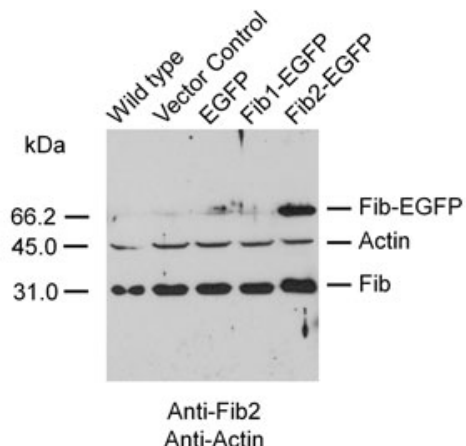

B

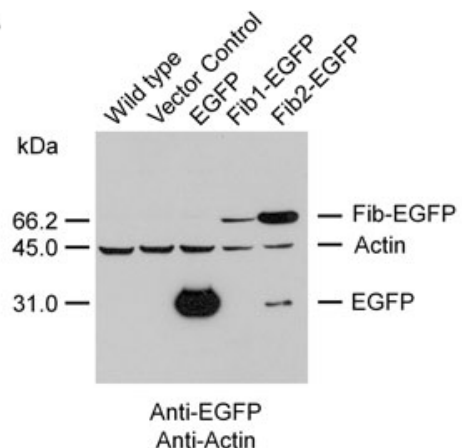

Anti-Actin

C

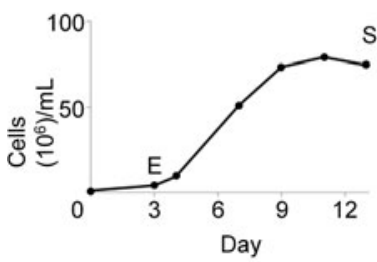

.
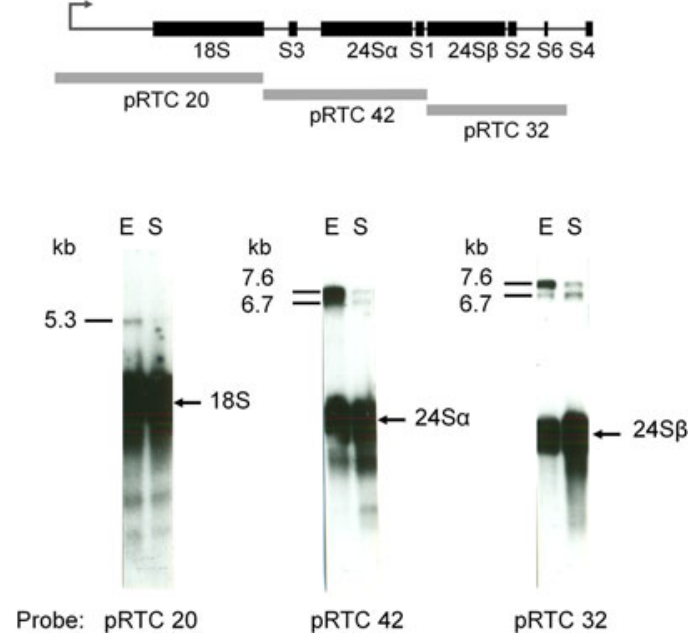

E S
pRTC 42
7.6 을

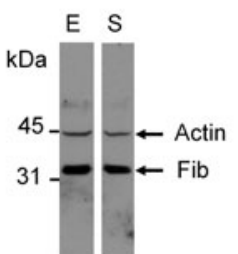

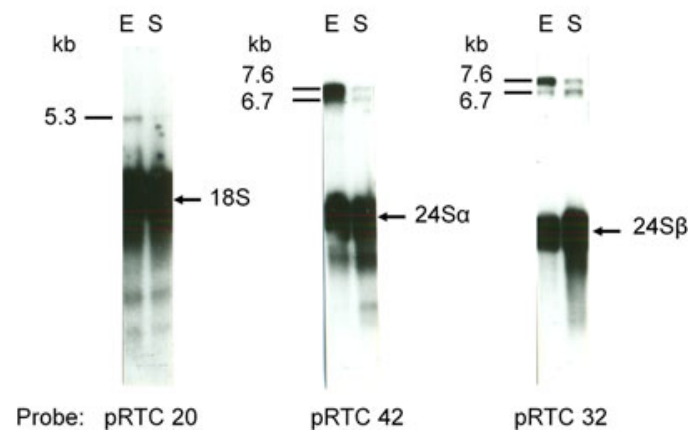

Fig. 3. Top: Expression of fibrillarin and EGFP-fibrillarin fusion proteins in epimastigotes. Protein extracts from either wild-type parasites or transfected epimastigoes (as indicated) were probed with either anti-TcFib2 antibodies (A) or antiEGFP antibodies (B). In addition, an anti-Tcactin polyclonal antibody was used as a protein loading reference in both blots (Cevallos et al. 2011). Bottom: Steady-state concentration of fibrillarin and pre-rRNAs in exponential and stationary epimastigotes. (C) Representative epimastigote growth curve, and comparative Western blot of total cellular proteins with both anti-actin (loading control) and anti-Fib2 homologous polyclonal antiserums. The protein size markers are broadrange SDS-PAGE standards from Bio Rad Laboratories (California). (D) Northern blots of total RNA probed with the indicated genomic clones with different regions of the rRNA gene transcription unit. Size estimation of molecular species was carried out using Gibco BRL RNA markers $(0 \cdot 2-9 \cdot 5 \mathrm{~kb})$. In both Western- and Northern blots, the same sample amount from the exponential phase or stationary cultures was loaded in the gels for their comparison.

RNA polymerase I activity. In this cited report, it was found that fibrillarin relocation correlated with a diminished abundance of pre-RNAs, particularly those comprising the $18 \mathrm{~S}$ mature rRNA. With such a reference, a potential difference in the steady-state concentration of pre-rRNAs between exponential and stationary T. cruzi epimastigotes was investigated by probing Northern blots of total RNA with genomic clones from the rDNA cistron. As expected for a ribosome-demanding physiological condition such as cellular growth, rRNA precursor molecules were more abundant in exponentially growing cells (E) than in stationary phase cells (S) (Fig. 3D).

\section{DISCUSSION}

To further study of nucleolar components in $T$. cruzi, we present our findings on the general characterization of fibrillarin expression and localization in exponentially growing and stationary epimastigotes in culture. Fibrillarin is annotated in the CL Brener hybrid reference strain as two pairs of haplotype genes. Their deduced amino acid sequences differ by approximately $35 \%$ (identity comparison). In spite of these dissimilarities, potential functional domains such as the GAR region and the MeTase domain can be recognized in both of the T. cruzi orthologues. Studies performed on A. pernix, $A$. thaliana and $S$. cerevisiae provide valuable descriptions of fibrillarin functional domains (Niewmierzycka and Clarke, 1999; Reichow et al. 2007; Rakitina et al. 2011; De Silva et al. 2012). Given that these features are observed in the deduced proteins from both T. cruzi fibrillarin genes, no obvious functional discrimination can be predicted for either of them.

Arabidopsis thaliana is a species with two fibrillarin genes that encode nearly identical proteins (Barneche et al. 2000). Potential differences in their 


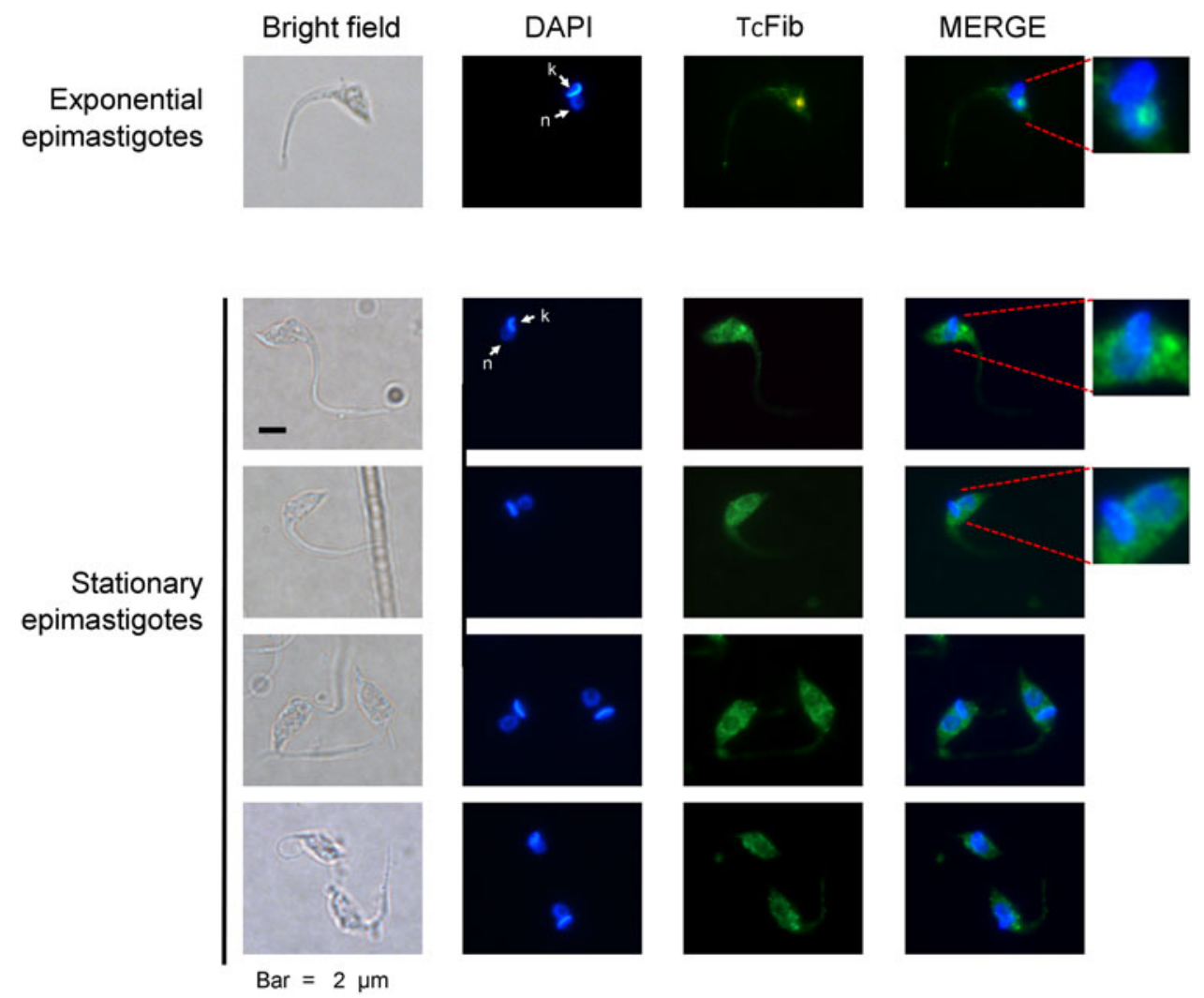

Fig. 4. Fibrillarin expression in epimastigotes. Epifluorescence images of epimastigotes after staining with anti-TcFib2 antibodies (green) and DAPI (blue). A selected image of growing epimastigotes is shown in the top panel, a collection of images of stationary cells is presented below. In some cases an amplified inset of the nuclear region (merged image) is presented to better illustrate the relation between fluorescent signals.

function have not been addressed other than that both proteins complement a NOP-1 null yeast mutant. Accessed genomes from trypanosomatids

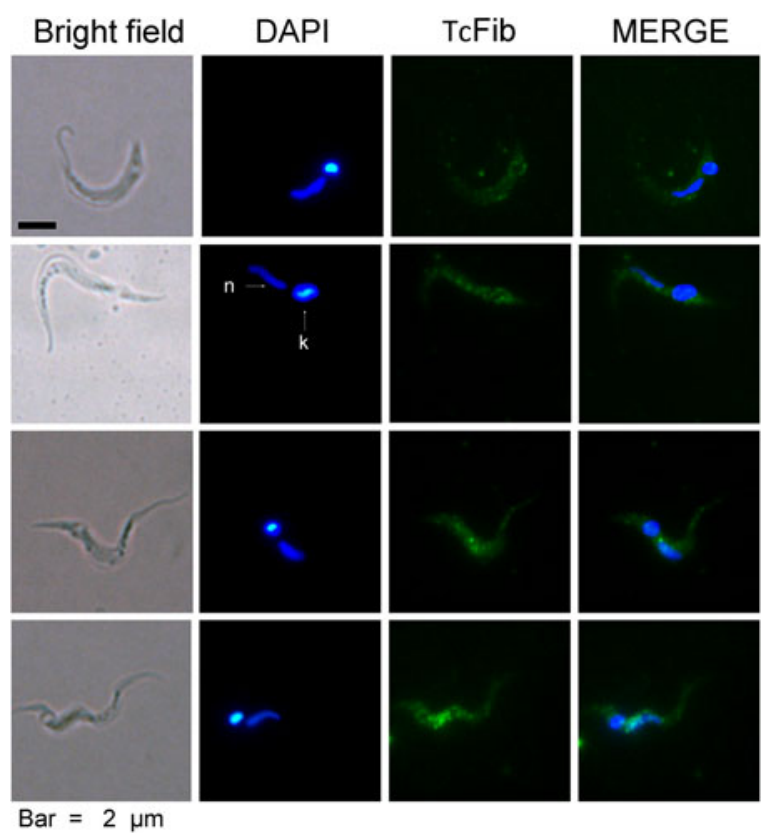

Fig. 5. Fibrillarin expression in metacyclic trypomastigotes. Epifluorescence images of trypomastigotes from cultures at the stationary phase after staining with anti-TcFib2 antibodies (green) and DAPI (blue). contain at least two annotated fibrillarin genes. The only experimental description of one gene has been done in T. brucei (Barth et al. 2008). In this study, NOP1 (accession number TriTryp: Tb927.10. 7500 ) was silenced by RNA interference. The associated phenotype was cellular lethality and rRNA processing and modification defects.

Data on two dissimilar fibrillarin genes in T. cruzi are presented here. Ectopic fibrillarin gene expression from plasmid vectors produced nucleolar location of fluorescent EGFP fusions for both TcFib1 and TcFib2 chimaeric genes. An equivalent finding was observed in $T$. brucei cells transfected with a NOP1-GFP fusion (Marchetti et al. 2000). In the present work, an antiserum could be raised against TcFib2 recombinant protein. This antibody recognized a nucleolar protein in exponentially growing epimastigotes. Potential linkage of its expression regulation to non-dividing conditions was addressed. It is to be noted that non-growing epimastigotes from the stationary phase harbour a nucleolus reduced in both size and granularity (Nepomuceno-Mejía et al. 2010). This type of cell expresses a similar amount of fibrillarin, at least TcFib2, compared with exponentially growing epimastigotes. Interestingly, most cells from aged cultures presented a granular cytoplasmic fluorescent signal, as did the metacyclic trypomastigotes that 
appear in these stressed cultures. Trypomastigotes are nucleolar-less cells as reported by Elias et al. 2001. A partial relocation of nucleolar fibrillarin to the cytoplasm after a low dose of actinomycin D exposure has been described in Xenopus kidney cells. This observation occurred in correlation with a reduction of pre-rRNA abundance, specifically those molecular species that produce the mature small subunit rRNA component (Rivera-Léon and Gerbi, 1997). The finding presented here of reduced rRNA precursor abundance in stationary T. cruzi cells is consistent with this early observation (Rivera-Léon and Gerbi, 1997). It can be then suggested that the intracellular location of fibrillarin in T. cruzi depends on physiological processes, yet to be determined.

\section{ACKNOWLEDGEMENTS}

We thank Juliana Herrera-López, Karla López-Pacheco and Patricia Espinosa-Cueto for technical assistance. We also acknowledge Evelyn Fein for her help in the very early stages of this work.

\section{FINANCIAL SUPPORT}

Ernesto Guerrero-Sánchez was a doctoral student from Programa de Doctorado en Ciencias Biomédicas, Universidad Nacional Autónoma de México (UNAM) and received fellowship 362997 from CONACyT. We also acknowledge financial support from Dirección General de Asuntos de Personal Académico, UNAM, through grant IN201014 (R.H.).

\section{REFERENCES}

Amiri, K. A. (1994). Fibrillarin-like proteins occur in the domain Archaea. Fournal of Bacteriology 176, 2124-2127.

Barneche, F., Steinmetz, F. and Echeverria, M. (2000). Fibrillarin genes encode both a conserved nucleolar protein and a novel small nucleolar RNA involved in ribosomal RNA methylation in Arabidopsis thaliana. The Fournal of Biological Chemistry 275, 27212-27220.

Barth, S., Shalem, B., Hury, A., Tkacz, I. D., Liang, X.-H., Uliel, S., Myslyuk, I., Doniger, T., Salmon-Divon, M., Unger, R. and Michaeli, S. (2008). Elucidating the role of C/D snoRNA in rRNA processing and modification in Trypanosoma brucei. Eukaryotic Cell 7, 86-101.

Camargo, E. P. (1964). Growth and differentiation in Trypanosoma cruzi. I. Origin of metacyclic trypanosomes in liquid media. Revista do Instituto de Medicina Tropical de São Paulo 6, 93-100.

Cevallos, A. M., Segura-Kato, Y. X., Merchant-Larios, H., ManningCela, R., Alberto Hernández-Osorio, L., Márquez-Dueñas, C., Ambrosio, J. R., Reynoso-Ducoing, O. and Hernández, R. (2011). Trypanosoma cruzi: multiple actin isovariants are observed along different developmental stages. Experimental Parasitology 127, 249-259.

Collins, T. J. (2007). Image J for microscopy. Biotechniques 43, 25-30.

De Silva, U., Zhou, Z. and Brown, B. A. (2012). Structure of Aeropyrum pernix fibrillarin in complex with natively bound S-adenosyl-L-methionine at $1.7 \AA$ resolution. Acta Crystallographica F, Structural Biology and Crystallization Communications 68, 854-859.

El-Sayed, N. M., Myler, P. J., Bartholomeu, D. C., Nilsson, D., Aggarwal, G., Tran, A.-N., Ghedin, E., Worthey, E. A., Delcher, A. L., Blandin, G., Westenberger, S. J., Caler, E., Cerqueira, G. C., Branche, C., Haas, B., Anupama, A., Arner, E., Aslund, L., Attipoe, P., Bontempi, E., Bringaud, F., Burton, P., Cadag, E., Campbell, D. A., Carrington, M., Crabtree, J., Darban, H., da
Silveira, J. F., de Jong, P., Edwards, K. et al. (2005). The genome sequence of Trypanosoma cruzi, etiologic agent of Chagas disease. Science (New York, NY) 309, 409-415.

Elias, M. C., Marques-Porto, R., Freymüller, E. and Schenkman, S. (2001). Transcription rate modulation through the Trypanosoma cruzi life cycle occurs in parallel with changes in nuclear organisation. Molecular and Biochemical Parasitology 112, 79-90.

Finn, R. D., Bateman, A., Clements, J., Coggill, P., Eberhardt, R. Y., Eddy, S. R., Heger, A., Hetherington, K., Holm, L., Mistry, J., Sonnhammer, E. L. L., Tate, J. and Punta, M. (2014). Pfam: the protein families database. Nucleic Acids Research 42 (Database issue), D222-D230.

Gerbi, S. A., Borovjagin, A. V., Ezrokhi, M. and Lange, T. S. (2001). Ribosome biogenesis: role of small nucleolar RNA in maturation of eukaryotic rRNA. Cold Spring Harbor Symposia on Quantitative Biology 66, 575-590. Hernández, R. and Cevallos, A. M. (2014). Ribosomal RNA gene transcription in trypanosomes. Parasitology Research 113, 2415-2424.

Hernández, R., Díaz-de Léon, F. and Castañeda, M. (1988) Molecular cloning and partial characterization of ribosomal RNA genes from Trypanosoma cruzi. Molecular and Biochemical Parasitology 27, 275-279.

Kelly, J. M., Ward, H. M., Miles, M. A. and Kendall, G. (1992). A shuttle vector which facilitates the expression of transfected genes in Trypanosoma cruzi and Leishmania. Nucleic Acids Research 20, 3963-3969. Lafontaine, D. L. and Tollervey, D. (2000). Synthesis and assembly of the box C + D small nucleolar RNPs. Molecular and Cellular Biology 20, 2650-2659.

Marchetti, M. A., Tschudi, C., Kwon, H., Wolin, S. L. and Ullu, E. (2000). Import of proteins into the trypanosome nucleus and their distribution at karyokinesis. Fournal of Cell Science 113, 899-906.

Martínez-Calvillo, S., López-Villaseñor, I. and Hernández, R. (1997). pRIBOTEX expression vector: a pTEX derivative for a rapid selection of Trypanosoma cruzi transfectants. Gene 199, 71-76.

Michaeli, S. (2012). rRNA Biogenesis in Trypanosomes. In RNA Metabolism in Trypanosomes (ed. Bindereif, A.), pp. 123-148. SpringerVerlag, Berlin, Heidelberg.

Nepomuceno-Mejía, T., Lara-Martínez, R., Cevallos, A. M., LópezVillaseñor, I., Jiménez-García, L. F. and Hernández, R. (2010). The Trypanosoma cruzi nucleolus: a morphometrical analysis of cultured epimastigotes in the exponential and stationary phases. FEMS Microbiology Letters 313, 41-46.

Niewmierzycka, A. and Clarke, S. (1999). S-Adenosylmethionine-dependent methylation in Saccharomyces cerevisiae. Identification of a novel protein arginine methyltransferase. The Fournal of Biological Chemistry 274, 814-824

Olson, M. O. J. and Dundr, M. (2005). The moving parts of the nucleolus. Histochemistry and Cell Biology 123, 203-216.

Rakitina, D. V., Taliansky, M., Brown, J. W. S. and Kalinina, N. O. (2011). Two RNA-binding sites in plant fibrillarin provide interactions with various RNA substrates. Nucleic Acids Research 39, 8869-8880.

Reichow, S. L., Hamma, T., Ferré-D'Amaré, A. R. and Varani, G. (2007). The structure and function of small nucleolar ribonucleoproteins. Nucleic Acids Research 35, 1452-1464.

Rivera-León, R. and Gerbi, S. A. (1997). Delocalization of some small nucleolar RNPs after actinomycin D treatment to deplete early prerRNAs. Chromosoma 105, 506-514.

Schimmang, T., Tollervey, D., Kern, H., Frank, R. and Hurt, E. C. (1989). A yeast nucleolar protein related to mammalian fibrillarin is associated with small nucleolar RNA and is essential for viability. The EMBO fournal 8, 4015-4024.

Sievers, F., Wilm, A., Dineen, D., Gibson, T. J., Karplus, K., Li, W., Lopez, R., McWilliam, H., Remmert, M., Söding, J., Thompson, J. D. and Higgins, D. G. (2011). Fast, scalable generation of high-quality protein multiple sequence alignments using Clustal Omega. Molecular Systems Biology 7, 539.

Tollervey, D., Lehtonen, H., Carmo-Fonseca, M. and Hurt, E. C. (1991). The small nucleolar RNP protein NOP1 (fibrillarin) is required for pre-rRNA processing in yeast. The EMBO Fournal 10, 573-583.

Wang, H., Boisvert, D., Kim, K. K., Kim, R. and Kim, S. H. (2000). Crystal structure of a fibrillarin homologue from Methanococcus jannaschii, a hyperthermophile, at 1.6 A resolution. The EMBO fournal 19, 317-323. Waterhouse, A. M., Procter, J. B., Martin, D. M. A., Clamp, M. and Barton, G. J. (2009). Jalview Version 2 -- a multiple sequence alignment editor and analysis workbench. Bioinformatics (Oxford, England) 25, 1189-1191. 